\title{
Respuesta productiva de vacas lactantes F1 Holstein x Gyr recibiendo ensilajes de maíz o sorgo como suplemento alimenticio en época seca
}

\author{
Productive response of lactating cows F1 Holstein x Gyr getting silage corn or \\ sorghum as food supplement in the dry season
}

\author{
GUEVARA S, CARMEN ${ }^{1 *}$ M.Sc, PATIÑO P, RENÉ ${ }^{1}$ Ph.D, MEJÍA M, CARLOS ${ }^{2}$ MVZ.
}

${ }^{1}$ Universidad de Sucre, Facultad Ciencias Agropecuarias. Sincelejo, Colombia. ${ }^{2}$ Profesional, Medicina Veterinaria y Zootecnia.

\section{Keywords:}

Corn silage; sorghum silage; grazing supplementation; tropical dry forest; dairy cattle in the tropics.

\begin{abstract}
The objective of this research was to evaluate the effect of supply on the performance of multiparous cows during the dry season corn silage or sorghum, in addition to the pasture. Twenty-seven multiparous cows ( $50 \%$ Holstein- $50 \%$ Gyr), during middle lactation, with $409 \pm 80 \mathrm{~kg}$ of BW, were used, to study feed intake, body weight variation and milk production. Animals were allocated to three treatments (T1: silage corn, T2: silage sorghum and T3: control group) for 30 days. All data were arranged in completely randomized design, the means were compared using orthogonal contrasts between treatments (control group vs the complement and the second contrast between maize and sorghum), using SPSS (v. 22) program, adopting 0.05 for Type I error. Cows fed silage how strategy during milking had significant effect on milk production $p<0.001$ weight gain of cows $p<0.001$ when compared with the control group. However, no significant differences for these two variables $p=0.986$ and $p=0.176$ respectively, were evident when corn silage or sorghum was supplemented. Supplementation increased milk production and improved weight gain of cows observed response of 2.2 liters/day and weight gain of $0.82 \mathrm{~kg} /$ day, respectively, over the control group.
\end{abstract}

\section{Resumen}

El objetivo de la presente investigación fue evaluar el efecto de la oferta de ensilaje de maíz o sorgo, como complemento a la pastura, sobre el desempeño productivo de vacas multíparas durante la época seca. Para el estudio de las variables consumo, ganancia media diaria y producción de leche se utilizaron 27 vacas multíparas F1 Holstein $\mathrm{x}$ Gyr, durante el segundo tercio de lactancia, con peso promedio inicial de $409 \pm 80 \mathrm{~kg}$, dispuestos en tres tratamientos (T1: ensilaje de maíz, T2: ensilaje de sorgo y T3: grupo control), durante un mes. Todos los datos fueron dispuestos en diseño completamente al azar, se compararon las medias entre tratamientos aplicando contrastes ortogonales (grupo control vs el complemento y el segundo contraste entre el maíz y el sorgo), utilizando el programa SPSS versión 22, adoptando 0,05 para el error Tipo I. Se pudo observar en este estudio que la estrategia de ofrecer un complemento alimenticio durante el ordeño, tuvo efecto significativo sobre la producción de leche $p<0,001$ y ganancia de peso de las vacas $p<0,001$ cuando comparado con el grupo control, sin embargo, no se evidenciaron diferencias significativas para estas dos variables $p=0,986$ y $p=0,176$ respectivamente, cuando se suplementó ensilaje de maíz o sorgo. La suplementación aumentó la producción de leche y mejoró la ganancia de peso de las vacas, observándose respuesta de 2,2 Litros/día y ganancia de peso de 0,82 kg/ día respectivamente, por encima del grupo control. 


\section{Introducción}

La alimentación básica en los sistemas de producción bovina en Colombia está constituida por forrajes de pastoreo, ya que representan una práctica económica con baja utilización de mano de obra. Sin embargo, la dependencia del pastoreo tiene como desventajas los efectos de las variaciones climáticas así como de las condiciones físicas y químicas del suelo. De esta manera, durante la época seca se presentan disminuciones importantes en la disponibilidad y calidad del forraje, que reduce la carga animal, los niveles productivos y las tasas de crecimiento (SÁNCHEZ, 2004).

Aunque se dispone de diferentes tecnologías de conservación para atenuar los efectos negativos de la estacionalidad forrajera, predomina el proceso de ensilaje por la facilidad de realizarlo en cualquier época del año y por su baja demanda en infraestructura (SÁNCHEZ, 2000). Sin embargo, todavía se presentan dificultades para su implementación en las fincas y su inclusión en las dietas (SÁNCHEZ y BÁEZ, 2002), especialmente por las características específicas de los forrajes tropicales disponibles para el proceso, los cuales presentan altos niveles de pared celular y deficiencias de los nutrientes necesarios para una fermentación óptima que pueden disminuir la calidad nutricional y el consumo voluntario del producto obtenido.

Por lo tanto, el ganadero se ve en la obligación de intensificar su producción especialmente en época seca, a fin de mantener la disponibilidad de forrajes de buena calidad bien sea para suministro en verde en el pastoreo o corte, o mediante el uso de alternativas de conservación, tales como: heno, henolaje y ensilaje. De esta manera, el uso de forrajes conservados ofrece la posibilidad de asegurar alimentos y almacenarlos para su empleo futuro, especialmente en períodos de escasez (NIETO y CAICEDO, 2012)

Según SUTTON (1989), los nutrientes ofrecidos en la dieta (proteínas, minerales, vitaminas, lípidos, carbohidratos) son los principales precursores de los constituyentes solidos de la leche. Por lo tanto, factores dietéticos, tales como el propio consumo, la cantidad de forraje en la ración, la proporción forraje-concentrado, la composición de los carbohidratos, la frecuencia con la que se ofrece el alimento, entre otros; contribuyen positiva o negativamente en la manutención de la concentración de grasa y demás componentes de la leche.

Además, En los últimos años se ha obtenido un conocimiento significativamente más preciso de la degradación de la fibra en el rumen, que ha permitido el desarrollo de sistemas de manejo alternativos para mejorar la calidad del producto (leche y/o carne) (GALINA et al., 2007). Como consecuencia, surgen alternativas alimenticias como el ensilaje de maíz y de sorgo, que además de permitir conservar el alimento durante la época seca, permiten aportar un nivel adecuado de fibra y energía a la dieta.

Hay que destacar que en Colombia, más de la mitad del maíz y otros cereales que se utilizan para la elaboración de concentrados animales, sobre todo para ganado bovino, son importados; por lo que es un sistema de alimentación costoso para el ganadero, convirtiéndose así el ensilaje en un modo de alimentación más económica que puede cumplir con los requerimientos nutricionales del animal, (MOLINA et al., 2004).

En atención a las anteriores consideraciones, el objetivo del presente trabajo fue evaluar el efecto de la oferta de ensilaje de maíz o sorgo, como complemento a la pastura, sobre el desempeño productivo de vacas multíparas durante la época seca.

\section{Materiales y Métodos}

Periodo experimental. Se llevó a cabo durante 30 días, entre enero y febrero de 2015 , en las instalaciones de la finca Villa Laura, ubicada en Sabana Larga, el municipio de Chinú - Córdoba, clasificado como bosque seco tropical, a una altura sobre el nivel del mar de 14 metros, temperatura promedio de $27,5{ }^{\circ} \mathrm{C}$, humedad relativa de $81 \%$ y precipitación promedio anual de 1340 $\mathrm{mm}$; con época de lluvias comprendida entre abril y noviembre, durante la cual se registra aproximadamente el $85 \%$ de la precipitación y época seca desde noviembre hasta mediados de abril. 
Recolección de la información: El estudio se llevó a cabo en un sistema de pastoreo rotacional, con 26 divisiones de aproximadamente una hectárea por potrero y dos días de ocupación, en un área establecida por Bothriochloa pertusa - Pasto Colosuana, dispuesto de comedero y bebedero.

Para el estudio de las variables consumo, ganancia media diaria y producción de leche se utilizaron 27 vacas multíparas F1 Holstein x Gyr, durante el segundo tercio de lactancia, con peso promedio inicial de $409 \pm 80 \mathrm{~kg}$, debidamente organizadas en tres tratamientos ( $\mathrm{T} 1$ : ensilaje de maíz, T2: ensilaje de sorgo y T3: grupo control) (Tabla 1). Además de agua, se les suministró sal mineralizada a voluntad al $4 \%$ de $P$.

Tabla 1. Composición química de las dietas.

\begin{tabular}{ccc}
\hline \multirow{2}{*}{ Componente (\%) } & \multicolumn{2}{c}{ Tratamientos } \\
\cline { 2 - 3 } & Ensilaje de maíz T1 & Ensilaje de sorgo T2 \\
\hline Materia seca & 26,4 & 25,3 \\
Proteína bruta & 8,03 & 9,75 \\
Extracto etéreo & 4,36 & 3,48 \\
Cenizas & 7,5 & 8,5 \\
\hline
\end{tabular}

Una vez cuantificado el peso corporal inicial de las unidades experimentales, producción de leche diaria y etapa de lactancia, se dio inicio al periodo experimental:

Todos los días cerca de las 4:30 am, las vacas pertenecientes a cada tratamiento fueron debidamente trasladadas del potrero al corral de ordeño, provisto de comederos y bebederos, cada lote fue separado y enseguida suministrado en todos los comederos sal mineralizada al $4 \% \mathrm{P}$, además, el T1 recibió $20 \mathrm{~kg} / \mathrm{animal} /$ día de ensilaje de maíz, $20 \mathrm{~kg} / \mathrm{animal} / \mathrm{día}$ de ensilaje de sorgo para el T2 y el grupo control (T3) no recibió ningún tipo suplemento.

Una vez finalizado el ordeño manual, todos los animales son trasladados nuevamente a la pradera.

El periodo experimental tuvo una duración de 30 días, de los cuales 20 días fueron de adaptación a las dietas experimentales y 10 de colecta de datos:

Consumo de alimento: Desde el día 1 hasta el día 15 del periodo experimental los animales recibieron $15 \mathrm{~kg} / \mathrm{animal} / \mathrm{día}$ de ensilaje de sorgo o maíz, a partir del día 16 hasta el día 30, ambos grupos pasaron a consumir $20 \mathrm{~kg} / \mathrm{animal} / \mathrm{día}$ de cada alimento.

Variación del peso vivo animal: En los tres tratamientos, la vaca y la cría se pesaron al inicio del experimento y en intervalos de 7 días, totalizando 4 registros durante los 30 días, con una báscula electrónica portátil, realizando pesajes individuales determinando la ganancia diaria de peso (GDP) con la siguiente fórmula: $\mathrm{GDP}=(\mathrm{P}$ final $-\mathrm{P}$ inicial $) /$ Número de días.

Variación de la producción de leche: Al inicio del experimento se midió la producción de leche individual, y a partir del día 21 hasta el día 30 del periodo experimental (momento en el que los animales estaban adaptados a las dietas experimentales), se registraron los datos de producción de leche/animal/día, totalizando 11 tomas de datos: uno al inicio y 10 al finalizar la investigación de campo.

Diseño experimental: Se utilizó un diseño completamente al azar en arreglos de medidas repetidas en el tiempo, con tres tratamientos y nueve repeticiones cada uno, totalizando 27 unidades experimentales. Para las variables: ganancia media diaria de las vacas $(\mathrm{kg})$, ganancia media diaria de las crías $(\mathrm{kg})$ y producción de leche (L/día), se tomó el día cero de experimento como una covariable y se compararon las medias entre tratamientos aplicando contrastes ortogonales; siendo uno de los contrastes el grupo control vs el complemento (ensilajes de maíz o sorgo), y el segundo contraste entre el maíz y el sorgo. Todos los datos fueron analizados utilizando el programa SPSS versión 22, adoptando 0,05 para el error Tipo I.

\section{Resultados}

Conforme el diseño experimental adoptado puede observarse en la Tabla 2 y Figura 1, que la inclusión o la estrategia alimenticia de ofrecer un tipo de alimento durante el ordeño, tuvo efecto significativo sobre la producción de leche $p<0,0001$ (ensilaje de maíz 5,99 Litros/día o ensilaje de sorgo 6,00 Litros/ día vs grupo control 3,63 Litros/día), sin embargo, se pudo constatar que no hubo diferencias significativas entre el uso de ensilaje de maíz o sorgo p=0,986. 
Tabla 2. Medias estimadas de producción de leche (PL), ganancia diaria de peso de las vacas (GDPV) y crías (GDPC), Error y P-Valor de vacas lactantes de la raza Gyr recibiendo ensilajes de maíz o sorgo como complemento alimenticio.

\begin{tabular}{|c|c|c|c|c|c|c|}
\hline \multirow{2}{*}{ Variables } & \multirow{2}{*}{ Control } & \multirow{2}{*}{ Ensilaje de maíz } & \multirow{2}{*}{ Ensilaje de sorgo } & \multirow{2}{*}{ Error } & \multicolumn{2}{|c|}{ p-valor } \\
\hline & & & & & Contraste 1 & Contraste 2 \\
\hline PL & 3,633 & 5,989 & 6,000 & 0,334 & $<0,0001$ & 0,986 \\
\hline GDPV & $-0,051$ & 0,820 & 0,651 & 0,830 & $<0,0001$ & 0,176 \\
\hline GDPC & 0,957 & 0,942 & 0,939 & 0,132 & 0,566 & 0,923 \\
\hline
\end{tabular}

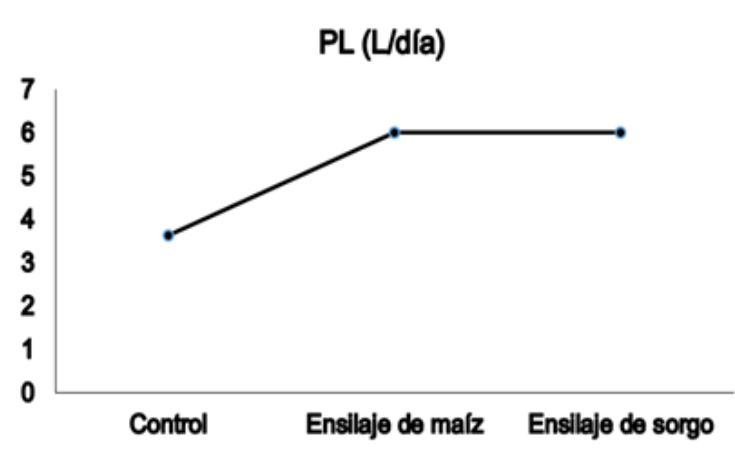

Figura 1. Medias estimadas de producción de leche de vacas lactantes de la raza Gyr recibiendo ensilajes de maíz o sorgo como complemento alimenticio (L/día).

También, se pudo observar el mismo comportamiento para la variable desempeño de las vacas (Tabla 2 y Figura 2), observándose efecto significativo $p<0,0001$ cuando se ofreció un complemento alimenticio (ensilaje de maíz 0,82 $\mathrm{kg} /$ día o ensilaje de sorgo $0,65 \mathrm{~kg} /$ día vs grupo control $-0,05 \mathrm{~kg} /$ día), lo que demuestra que durante la época seca, la no suplementación de los animales puede acarrear en pérdidas de tipo productivo y por consiguiente de tipo económica. No obstante, cuando se comparó la influencia del tipo de complemento alimenticio sobre la ganancia de peso, se pudo observar que no fue significativa $p=0,176$.

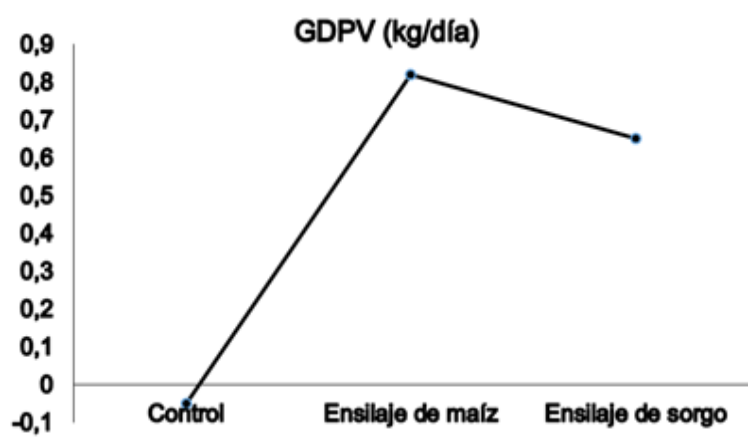

Figura 2. Medias estimadas de ganancia diaria de peso de vacas lactantes de la raza Gyr recibiendo ensilajes de maíz o sorgo como complemento alimenticio (kg/día).
Sin embargo, nótese que el desempeño de las crías (Tabla 2 y Figura 3 ) no fue afectado por la estrategia alimenticia de ofrecer o no un complemento alimento durante el ordeño $(p>0,05)$, en media, las crías ganaron $0,95 \mathrm{~kg} /$ día de peso vivo.

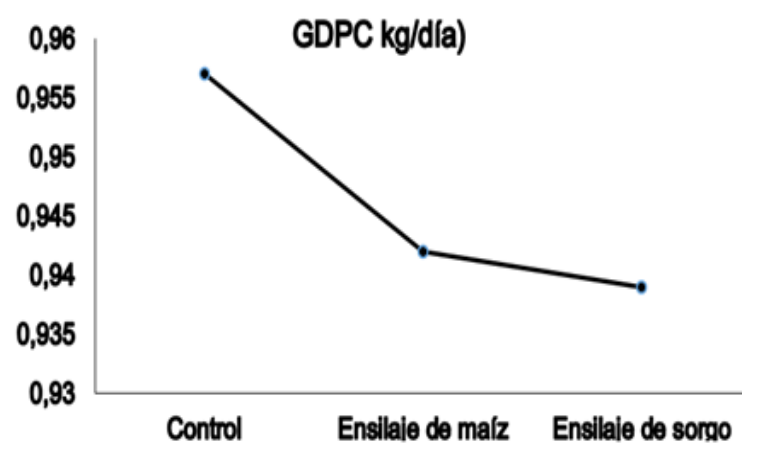

Figura 3. Medias estimadas de ganancia diaria de peso de terneros lactantes cuyas madres recibieron ensilajes de maíz o sorgo como complemento alimenticio (kg/día).

\section{Discusión}

La inclusión o la estrategia alimenticia de ofrecer ensilaje de maíz o sorgo durante el ordeño, tuvo efecto significativo sobre la producción de leche (5,99 y 6,00 L/día respectivamente), cuando comparado con el grupo que no recibió ningún complemento alimenticio (3,63 L/día) durante el periodo experimental; no obstante, no se observaron diferencias significativas sobre esta variable entre ambos complementos alimenticios, pudiéndose ofrecer alguno de los dos durante la época seca, permitiendo con ello el mantenimiento o aumento de la producción láctea durante esta época. Así mismo, ACOSTA (2006), comparando el efecto del ensilaje de grano húmedo de maíz, sorgo y trigo para producción de leche; no observó diferencias de consideración sobre la producción de leche entre la inclusión de estos materiales, particularmente a niveles medios de suplementación. 
De igual manera, se observó variación del peso de los animales no suplementados $(-0,05 \mathrm{~kg} /$ día) vs los animales que recibieron ensilaje de maíz $(0,820 \mathrm{~kg} / \mathrm{día})$ o sorgo $(0,651 \mathrm{~kg} / \mathrm{día})$. La elevada variación negativa para el tratamiento que no recibió ningún complemento alimenticio, se debió a la posible movilización de las reservas corporales por el animal en la tentativa de suplir las deficiencias nutricionales ocasionadas por el menor consumo de alimento debido a la baja calidad de los pastos en época seca. Esta variación del peso corporal fue observada por COSTA et al. (2005), al reemplazar el ensilaje de maíz en proporción de $60 \%$ en la dieta por caña de azúcar en las proporciones 60, 50 y $40 \%$ respectivamente, verificando que la dieta con mayor inclusión de caña de azúcar presentó la mayor variación negativa del peso corporal $-0,562$ kg/día, ocasionado por el menor consumo de alimento debido a la baja calidad composicional de la dieta y el exceso de fibra.

Sin embargo, la ausencia de diferencia significativa entre el uso de ensilaje de maíz o ensilaje de sorgo puede estar relacionado al número de animales evaluados, debido a las características metabólicas de cada animal, una vez que, según estudio realizados por DADO y ALLEN (1994) el número mínimo de vacas necesarias para obtener diferencias significativas entre dos tratamientos para variables como tiempo de alimentación, rumia y masticación total, serian 164, 52 y 44 animales, respectivamente en un delineamiento completamente al azar.

En un estudio comparativo entre el grano de maíz y el grano de sorgo con bajo y alto contenido de taninos, en dietas de animales para ceba, realizado por RIFFEL en 2004, cuyos tratamientos eran: a) $70 \%$ grano de maíz + 30\% (mezcla de sojilla + harina de soja + triguillo + núcleo vitamínico mineral); b) $70 \%$ grano de sorgo con bajo nivel de tanino (3.5 gramos/kg de MS) + $30 \%$ (mezcla de sojilla + harina de soja + triguillo + núcleo vitamínico mineral) y c) $70 \%$ grano de sorgo con alto nivel de tanino (9.5 gramos $/ \mathrm{kg}$ de MS) $+30 \%$ (mezcla de sojilla + harina de soja + triguillo + núcleo vitamínico mineral), observó ganancias diarias de peso de 1,69 kg/día, 1,18 kg/ día y 1,54 kg/día, para el grupo que recibió maíz grano, sorgo con bajo nivel de taninos y sorgo con alto nivel de taninos respectivamente, estos datos son superiores a los observados en este estudio, aunque cabe resaltar que el comportamiento y superioridad de las dietas con ensilaje de maíz $(0,82 \mathrm{~kg} /$ día $)$ permitieron mejores ganancias de peso cuando comparados con el ensilaje de sorgo $(0,65 \mathrm{~kg} / \mathrm{día})$.

En otro estudio, VALVASORI et al. (1998) evaluaron el desempeño de terneros holandeses alimentados con ensilaje de sorgo granífero y ensilaje de caña de azúcar suplementado con harina de algodón. Estos pudieron constatar la superioridad del ensilaje de sorgo frente al ensilaje de caña; siendo el ensilaje de sorgo quien presentó valores mayores de ganancia de peso diaria $(0,601 \mathrm{~kg} / \mathrm{día})$ similar a lo observado en este estudio, para la variable desempeño.

ROJAS y MARÍQUEZ (2001) compararon el efecto del ensilaje de maíz y ensilaje de trigo en la engorda invernal de novillos, estos observaron ganancias medias de diarias de 0,946 kg/día para el grupo alimentado con ensilaje de maíz, este resultado es superior a lo reportado por este estudio, pues el sexo y el estado fisiológico de los animales era diferente.

\section{Conclusiones}

La utilización de ensilajes dentro de la dieta de vacas en etapa de lactancia durante la época seca produjo una mejora en cuanto a las variables producción de leche, observándose respuesta de 2,2 Litros/día por encima del grupo control.

Los complementos alimenticios que se ofrecieron, no solo mantuvieron el peso de las vacas durante la época de verano sino que provocaron mejoras en la ganancia de peso de 0,82 y 0,65 kg/día cuando se utilizó ensilaje de maíz y ensilaje de sorgo respectivamente.

La no suplementación en época seca causa pérdidas de condición corporal, lo que a su vez se ve reflejado en el descenso de la producción de leche. 


\section{Referencias}

ACOSTA, Y.M. 2006. Programa Nacional de Lechería. Ingeniería Agronomica. Uruguay.

COSTA, M.G.; CAMPOS, J.M.S.; VALADARES FILHO, S.C. et al. 2005. Desempenho Produtivo de Vacas Leiteiras Alimentadas com Diferentes Proporções de Cana-de-Açúcar e Concentrado ou Silagem de Milho na Dieta. Revista Brasileira de Zootecnia 34 (6):2437-2445.

DADO, R.G.; ALLEN, M.S. 1994. Variation in and relationships among feeding, chewing, and drinking variables for lactating dairy cows. Journal of Dairy Science 77:132-144.

GALINA, M.A.; OSNAYA, F.; CUCHILLO, H.M., HAENLEIN, GFW. 2007. Cheese quality from milk of grazing or indoor fed Zebu cows and Alpine crossbred goats. Small Ruminant Research 71: 264-272.

MOLINA, A.M.G.; ROA, L.B.; ALZATE, S.R.; DE LEON, J.G.S.; ARANGO, A.F.B. 2004. Ensilaje como fuente de alimentación para el ganado. Revista lasallista de investigación 1 (1):66-71.

NIETO, C.; CAICEDO, C. 2012. Análisis reflexivo sobre el Desarrollo Agropecuario Sostenible en la Amazonía Ecuatoriana. INIAP - EECA. Publicación Miscelánea No. 405. Joya de los Sachas. Ecuador. $102 p$

RIFFEL, S. 2004. Trabajo de tesis de posgrado: ingeniería agronómica (inta balcarce 2004, Buenos Aires, Argentina).

ROJAS, C.; MANRÍQUEZ, M. 2001. Comparación de ensilaje de trigo y de maíz en la engorda invernal de novillos. Revista Agricultura Técnica 61 (4):444-451.

SÁNCHEZ L. 2000. Alterativas de utilización de ensilajes en explotaciones lecheras de trópico alto. Carta Fedegan 64:44-51. Bogotá, Colombia.

SÁNCHEZ, L. 2004. Nuevas estrategias para conservación de forrajes en el trópico. Primera Reunión de la Red Temática de Recursos Forrajeros. CORPOICA, Tibaitatá. Memorias. Mosquera, junio 2004. 15 p.

SÁNCHEZ, L.; BÁEZ, F. 2002. Conservación de forrajes en sistemas de producción bovina del trópico de altura. En: Alterativas tecnológicas para la producción competitiva de leche y carne en el trópico bajo. Plan de Modernización de la Ganadería Bovina Colombiana. CORPOICA, MADR, FEDEGAN, Fondo Nacional del Ganado. p. 17

SUTTON, J.D. 1989. Altering Milk Composition by Feeding. Journal of Dairy Science 72: 2801-2814.

VALVASORI, E.; LUCCI, C.S.; PIRES, F.L. et al. 1998. Desempenho de bezerros recebendo silagens de sorgo ou de cana-de-açúcar como únicos alimentos volumosos. Brazilian Journal of Veterinary Research and Animal Science 35 (5):229-232. 